\title{
Self-reported psychological disorders among the mothers of children with an autism spectrum disorder, type 1 diabetes mellitus, and typically developed children
}

Ahmed Malalla Al-Ansari ( $\nabla$ ahmedm.alansari@gmail.com )

Ministry of Health https://orcid.org/0000-0001-9577-6298

Mohammed Ismail Janahi

Arabian Gulf University College of Medicine and Medical Science

Abdulrahman Jamal AlTourah

College of Medicine and Medical Sciences, Arabian Gulf University

Haitham Ali Jahrami

Ministry of Health

Mansour Bin Rajab

Ministry of Health

\section{Research}

Keywords: ASD, diabetes, DASS-21, PSS-14, Bahrain

Posted Date: September 2nd, 2020

DOI: https://doi.org/10.21203/rs.3.rs-66198/v1

License: (c) (1) This work is licensed under a Creative Commons Attribution 4.0 International License.

Read Full License

Version of Record: A version of this preprint was published at Journal of Neurodevelopmental Disorders on May 22nd, 2021. See the published version at https://doi.org/10.1186/s11689-021-09369-y. 


\section{Abstract}

Objectives

To assess the prevalence of symptoms of depression, anxiety, and stress among mothers of children of autism spectrum disorders (ASD), type 1 diabetes, and typically developed (TD).

Method. The study sample comprised mothers of children with ASD ( Group 1; $n=116$ ), diabetes mellitus (Group 2; $\mathrm{n}=43$ ), and a control group of mothers with TD children.

Results

Both groups 1 and 2 had higher scores of depression (37.86), anxiety (4.58), and stress (29.81) in DASS21 in comparison to control $(P=0.015)$. On PSS-14 The mean score on PSS-14 were: group 1 (27.61), group $2(28.63)$ and control $(25.87)(p=0.004)$. Group 1 scored higher scores for depression domain $(p=$ $0.046)$ whereas group 2 for anxiety domain $(p=0.034)$ and stress domain $(p=0.009)$ in comparison to TD group on DASS-21.

Conclusion

Mothers of children with ASD should be assessed for the presence of depression following diagnosis. Mothers of children with type 1 diabetes required careful monitoring for the effects of anxiety and stress on their mental health, therefore their ability to cope with a management plan.

\section{Introduction}

Several studies have reported that families of children with autism spectrum disorder (ASD) experience more parenting stress than families of typically developing (TD) children or those diagnosed with other disabilities $(1,2,3,4)$. From these studies, an emerging trend suggests that mothers of children with ASD exhibit higher levels of depression, anxiety, and stress than the general population.

Several international studies support what has been reported previously. A study in Italy assessed the parental burden in families of children with ASD compared to a control group of parents with children who were diagnosed with type 1 diabetes mellitus, and parents with children diagnosed with Down syndrome. The parents of children with ASD reported higher objective and subjective burden, more frequent psychological distress, and lower social support. Moreover, mothers reported greater subjective burden than fathers did (5). A study in the Netherlands found that both parents reported more stress when parenting with their child with ASD and/or ADHD than while parenting with the unaffected sibling; they also experienced more stress than the general population (6). In the United States, a multistate-wide study reported that parents of toddlers with ASD demonstrated increased parenting-related stress compared with parents of toddlers with developmental delay and typically developed children. However, psychological distress did not differ significantly between the groups (7). Although parental stress, anxiety, and depression were reported significantly higher in parents of children with ASD than in parents 
of TD children, a study in the United Kingdom found that parent-reported distress, anxiety, depression, and health were not correlated with physiological measures, with the exception that parents of children with ASD had significantly lower cortisol levels 30 min after waking(8).

Studies that addressed the prevalence of depression, anxiety, and stress among mothers of children with diabetes were not conclusive and revealed mixed results. Parents of children with type-1 diabetes have a higher expression of anxiety and depressive feelings compared with parents of children without diabetes $(9,10,11,12)$. All subjects in the above studies included both parents. A study by Hauenstein several years ago (13), did not find a difference in a group of mothers regarding their overall stress. The degree of anxiety and stress was not only high in the period following diagnosis but worries continued for a longer period due to the illness chronic nature (14).

In the EMRO region, studies conducted revealed similar findings. One study in Oman revealed that caregivers of children with ASD carry a greater risk of depression and stress than caregivers of children with intellectual disabilities, along with an even greater risk when compared to the general population (3). A study in Saudi Arabia found a mean score of depression and anxiety that was significantly higher in a sample of parents of children with ASD when compared to a control consisting of parents of TD children (4). studies from Iran noted that the most common psychiatric conditions among parents of children with ASD were poor mental health "melancholic personality, persistent depression, and negativism" $(16,17)$. A prospective study in Kuwait found that mothers of children with ASD had higher levels of parentingrelated stress and psychological distress when compared to a control, the study also reported no significant difference between fathers of children with ASD and fathers from the general population (17). In Bahrain, mothers were found to be negatively impacted by having children with ASD. The sample studied was found to have more physical and mental health issues, along with a decreased quality of life, when compared to the general population. Mothers also reported more physiological and environmental problems than mothers of intellectually disabled children and typically developed children $(2,18)$. We could not trace any study addressing the mothers of diabetic children's mental health locally.

The goal of this study is to identify any significant differences in depression, anxiety, and stress among mothers of children with ASD when compared to mothers of children with another chronic illness, diabetes, and mothers of typically developed (TD) children.

\section{Method}

Design: A case-control study.

Sample. Cases comprised mothers of children, age between 3-18 years, with ASD diagnosis (group 1) and type 1 diabetes (group 2). The diagnosis of ASD was reached clinically using Modified-Checklist for Autism in Toddlers (M-CHAT), Diagnostic Statistical Manual for Mental Disorder 5 definition (DSM-5). Childhood Autism Rating Scale (C.A.R.S) and Autism Diagnostic Observation Schedule (ADOS) in certain cases, at the Child Psychiatric Unit, Psychiatric Hospital, Kingdom of Bahrain (19). The number of cases identification initially was 172 which later reduced to 126 as 46 cases were excluded due to non-eligibility. 
Inclusion criteria for cases were mothers who had one child age 3-18 years with either ASD or type 1 diabetes. Exclusion criteria were mothers who had other children with a disability or chronic illness defined as continuous symptoms for more than six months, mothers who had legal or marital or financial difficulties, mental health diagnoses, or chronic physical problems in the past month before the initial interview, were excluded. Seventy-seven mothers of children diagnosed as Type 1 diabetes were recruited from the Pediatric department, Outpatient Clinic, Salmaniya Medical complex, which later reduced to 43 according to the same exclusion criteria. All cases attending the Autism centers and the Diabetes clinic between June 15 to October 15, 2019, were recruited as convenience sampling.

General population control: Mothers from the general population recruited randomly that matched groups 1 and 2 both in the mean age of child and family size. The Control group included mothers who had a child with any neurodevelopmental disorder or chronic illness. A chronic illness is defined as an illness that lasted over six months continuously and is known to have an impact on the mother's mental health. The same other exclusion criteria for group 1 and 2 cases were applied to the control group.

Procedures.

Members of the research team (A.T. and M.J.) contacted all eligible cases and control by electronic means. Sixty from group 1 were interviewed face- to- face due to lack of computer literacy. All mothers in groups 1, 2, and the control group completed both the DASS-21 and PSS-14 (Arabic versions) questionnaires. Mothers were informed verbally of nature and the objectives of the research and their approval to participate in the study was obtained. It was explained to all participants that enrolment in the study is voluntary and will not affect the services provided to them and their children. Ethical approval was obtained from the Research Committee, Secondary care, Ministry of Health, Kingdom of Bahrain.

Instruments:

1. A datasheet to record the child's and mother's age, employment, and education.

2. An Arabic version of DASS-21 is a standard assessment tool for depression, anxiety, and stress symptoms with each of the three dimensions focused as several items. These items direct the recipient to only describe their experiences during the last one weak. This tool has been reported to have an acceptable validity for stress $(r=0.74)$ and anxiety $(r=0.81)$ when compared to the Beck Depression inventory (20).

3. Arabic Version, Perceived Stress Scale (PSS), PSS is the most widely used psychological instrument for the measurement of one's perception of their stress. For, PSS, recipients were asked to describe their thoughts and feelings during the last month (21).

Data Analysis. Data were entered into SPSS version 26. Descriptive statistics were summarized for the demographic characteristics and outcome measure. The mean and standard deviation (SD) was reported for continuous variables and applicable counts and percentages were reported for categorical variables. Pearson Chi-square or Fisher's exact test and independent-sample test was used to investigate the 
differences among the groups. Odds ratio and their respective confidence intervals were also determined for DASS-21 severity of stress, anxiety, and depression between groups 1 and 2 and TD-control.

\section{Results}

Table 1 shows the demography of cases in groups 1 and 2 and the control group. There were no differences as far as to mean child age, the mean number of children, and the mother's age group (31-60 $y)$, between the groups. There was no difference between the diabetic group and control in terms of education but mothers in the control were employed significantly more than the diabetes group. On the other hand, ASD group mothers were significantly less educated and unemployed in comparison to the TD-control group.

Table 2 shows the mean score of DASS-21 and PSS-14 among group 2 and control. Total DASS-21 scores were higher among case 2 compared to community control ( 41.58 vs $29.81-P=0.019)$. DASS-21 subscale means a score of depression and stress was higher among group 2 compared to control but it did not reach a significant level. According to PSS-14, the mean score was significantly higher among group 2 compared to control (28.63 vs $25.87 \mathrm{P}=0.003)$

Table 3 shows the mean score of DASS-21, PSS-14 among mothers of ASD (group 1), and diabetes group (group 2). On DASS-21, ASD group scores were higher for depression and stress compared to the diabetes group while the diabetic group scored more for anxiety symptoms $(\mathrm{OR}=1.742,95 \% \mathrm{Cl}=0.856$ $3.444, P=0.642$ ). The difference did not reach a significant level, on PSS-14 the diabetic group scored more stress compared to the ASD group but again the difference was not significant (28.63 vs 27.61 $\mathrm{P}=0.245)$. DASS-21 total scores were higher for group 2 (41.58) compared to group 1 (37.86) but it did not reach a significant level.

Table 4 shows the mean scores among the 3 groups by DASS-21 and PSS- 14 .

The DASS total score was significantly higher among group 2, group 1 than the control group, respectively $(41.58,37.86,29.81, p=0.015)$. Depressive symptoms were prevalent among group 1 in comparison to group 2 and control $p=0.042$, while anxiety and stress symptoms were reported higher among group 2 than case 1 and control ( $p=0.007$ and 0.030 , respectively. On PSS-14, group 2 scored 28.81 more than group $1,27.61$, and control, 25.87 $\mathrm{P}=0.002$.

Table 5 shows the prevalence of depression, anxiety, and stress among group 1, group 2, and control. Prevalence of depression was more among group 1 in comparison to group 2 and control, 57.1, 46.5, and 43.1 respectively $(p=0.046)$. Anxiety and stress were prevalent among group 2 in comparison to group 1 and control $p=0.034, p=0.009$ respectively.

\section{Discussion}


The findings in this study support the initial hypothesis and other previous reports $(3,5)$ that depression, anxiety, and stress are higher among mothers of children of ASD and type 1 diabetes in comparison to mothers of TD children. The impact of having chronic illness whether it is ASD or diabetes in this study on mother's health varies: mothers of children with ASD reported more depressive symptoms while mothers of children with diabetes presented with anxiety and stress symptoms much more than mothers of children with ASD and TD. Mothers of children with ASD developed more depressive symptoms as they lost the perfect child in their minds following the delivery of normal children. It is a known fact that depression may follow the death of relatives or friends while stress follows other types of stressful events (22). On the other hand, mothers of children with diabetes have daily worries regarding their children's blood sugar level and what could adversely impact that level. These worries continue to operate for a long-time due to the chronic nature of the illness and according to the child's developmental tasks and challenges of growing-up (23). Thus, the persistent feeling of anxiety and worries that mothers of children with diabetes exceed that present in mothers of ASD and TD. The level of depression, anxiety, and depression among mothers of children with diabetes exceed that reported by Weibe et al (25) and slightly less than the rates mentioned by Streisand et al (10). The caregiver strain stemming from anxiety about blood glucose monitoring is inversely related to adherence to management protocol in adolescents (24, 25).

The difference in employment rates among mothers of the 3 groups is because many mothers of TD groups were recruited from the participating centers staff, also many mothers of cases 1 and 2 choose to retire in order to provide better care to their children. The other explanation that mothers of TD groups were more educated and therefore had better chances to find jobs.

The assessment of parents' mental health, especially of mothers, during case management of ASD and children with diabetes, is critical. It has been shown that high levels of life stress are risk factors for poor health outcomes for both parents and children. $(26,27)$.

\section{Study limitations:}

The gathered information was drawn from a questionnaire, a method known to have a problem of subjectivity. The mothers were not subjected to any structured clinical interview to establish a reliable and valid diagnosis of depression or anxiety. The degree of social impairment of children was not evaluated in this study. Severe social impairment and the presence of behavioral problems among children will add to the mother's burden and their ability to provide adequate care. Furthermore, the socio-economic background was not exactly the same among the 3 groups. Mothers of children with TD were more educated and working compared to ASD and diabetes groups. One might speculate about the effect of these differences on mothers coping mechanisms and invariably the level of anxiety and stress. We did not address the duration since the diagnosis as a confounding variable due to the limited sample size. Future study designs should address these limitations and also include both parents. 
Clinical implications. The parent's mental health especially among mothers is an important determinant of better ability to cope with the burden of taking care of difficult children, in particular, those with ASD and diabetes. In our clinical service context, medical services provided to mothers of children with ASD and diabetes lack a specific provision for individual counseling and support during the process of diagnosis and long-term management. This drawback is probably related to insufficiently trained personnel and the absence of a well-designed strategic follow- up plan. Many clinicians feel their job is to establish the diagnosis and prescribe appropriate medication. They fail to give enough attention to the mother's feelings of depression and worries. We suggest that checking mothers/caregiver's mental health periodically should be a part of any monitoring process. The first step in their direction is to establish special separate services for both ASD and children with diabetes.

Summary and conclusion. In a case-control study, where cases were mothers of children of ASD and diabetes, and the control from the general population, the prevalence of depression, anxiety and stress were estimated using DASS-21 and PSS-14 instruments. Mothers of children with either ASD or diabetes scored higher on anxiety, depression, and stress in comparison to TD; however, the pattern varied between ASD and diabetes groups. Mothers of ASD showed more depressive symptoms while mothers of children with diabetes reported a higher prevalence of anxiety and stress. Follow up monitoring of children with ASD and diabetes should include ways of reducing depression and anxiety among mothers to equip them with a more stable mental health status.

\section{Declarations}

\section{Ethical Approval and Consent to participate}

From research committee secondary care, Ministry of Health, Bahrain.

\section{Consent for publication}

Approved

\section{Availability of supporting data}

Nil.

\section{Competing interests}

Nil.

\section{Funding}

Nil.

\section{Acknowledgements}

Not applicable. 
Authors' information

Not applicable.

\section{Conflict of Interest}

The authors of this study have no conflict of interest to declare. This work did not receive any financial support at any stage.

\section{Authors Contribution}

A.A: Conception, design, interpretation of data, drafting, revising the article, and final approval of the manuscript.

M.J: Acquisition of data, analysis, and final approval of the manuscript.

A.T: Conception, acquisition of data, analysis, interpretation of data, revising the article, final approval.

H.J: Conception, design, analysis, revising the article, and final approval of the manuscript.

M.R: Conception, design, and final approval of the manuscript.

\section{References}

1. Hayes SA, Watson SL. The Impact of Parenting Stress: A Meta-analysis of Studies Comparing the Experience of Parenting Stress in Parents of Children with and Without Autism Spectrum Disorder. J Autism Dev Disord. 2012;43(3):629-42.

2. Ansari AMA, Jahrami HA. Health of Mothers of Children with Autism Spectrum Disorders and Intellectual Disability. Bahrain Med Bull. 2018;40(3):157-61.

3. Al-Farsi O, Al-Farsi Y, Al-Sharbati M, et al. Stress, anxiety, and depression among parents of children with autism spectrum disorder in Oman: a case-control study. Neuropsychiatric Disease Treatment. 2016;12:1943-51.

4. Almansour MA, Alateeq MA, Alzahrani MK, et al. Depression and anxiety among parents and caregivers of autistic spectral disorder children. Neurosciences (Riyadh). 2013 Jan;18(1):58-63.

5. Picardi A, Gigantesco A, Tarolla E, et al. Parental Burden and its Correlates in Families of Children with Autism Spectrum Disorder: A Multicentre Study with Two Comparison Groups. Clinical Practice Epidemiology in Mental Health. 2018;14(1):143-76.

6. Steijn DJV, Oerlemans AM, Aken MAGV, et al. The Reciprocal Relationship of ASD, ADHD, Depressive Symptoms and Stress in Parents of Children with ASD and/or ADHD. J Autism Dev Disord. 20130ct;44(5):1064-76.

7. Estes A, Olson E, Sullivan K, et al. Parenting-related stress and psychological distress in mothers of toddlers with autism spectrum disorders. Brain Develop. 2013;35(2):133-8. 
8. Padden C, James JE. Stress among Parents of Children with and without Autism Spectrum Disorder: A Comparison Involving Physiological Indicators and Parent Self-Reports. Journal of Developmental Physical Disabilities. 2017;29(4):567-86.

9. Yacoob H, Ali Khan L, et al. Diabetes Mellitus in children and its effect on caregiver's mental health. Cureus. 2018;10(4):e2409. DOI 10.77.59/cureus.2409.

10. Streisland R, Mackey ER, Elliot BM, et al. Parental anxiety and depression associated with caring for a child newly diagnosed with type 1 diabetes: oppurtunities for education and counseling. Patient education counseling-. 2008;73:333-8.

11. Jasper SS, Linsky R, Grey M.Coping and psycholohical distress in mothers of adolscents with type 1 diabtes. Mateir child health J DOI.10.1007/s L0995-013-1239-4.

12. Whittemore RJasers, Chao A, Jang M, Corey M. Psychological experience of parents of children with type 1 diabetes: a systematic-mixed-study review. Diabetes Educ. 2012,38:562 - 79 DOI.10.1177/0145721712445216.

13. Hauenstien EJ, Marvin RS, Synder AL, Clark WL. Stress on parents of children with diabetes mellitus. Diabetes care 1989 1;12(1):18-23.

14. Harsh A, Mac Manus F, Kennedy P, Edge J. Anxiety depressive and post-traumatic stress symptoms in mothers of children with type 1 diabetes. Journal of traumatic stress. 2007;20(5):88-91.

15. Amiri S, Ranjbar F, Hatami R, et al. Psychopathology of the Parents of Autistic Children Based on the Clinical Personality Disorders. Annals of Psychiatry Treatment. 2016Nov;1(1):001-5.

16. Riahi F, Izadi S. Comparison between the mental health of mothers of children with autism and control group. Iran J Psychiatry Behav Sci. 2012;6(2):91-5.

17. Fido A, Saad SA. Psychological effects of parenting children with autism prospective study in Kuwait. Open Journal of Psychiatry. 2013;03(02):5-10.

18. Ansari AMA, Jahrami HA, Hafedh RG, et al. A Comparison of the mental health, quality of life, and general functioning of mothers of young children and adolescents with autism. Journal of the Bahrain Medical Society. 2018;30(2):17-24.

19. American Psychiatric Association. Diagnostic and Statistical Manual of Mental Disorders: Diagnostic and Statistical Manual of Mental Disorders, Fifth Edition. Arlington, VA: American Psychiatric Association, 2013.

20. Taouk M, Lovibond PF, Laube R. Psychometric properties of an Arabic version of the depression anxiety stress scales (DASS-21) Report for New South Wales transcultural mental health center, Cumberland hospital; Sydney:2001.

21. Linn MW. Modifiers and perceived stress scale. journal of consulting clinical psychology. 1986;54(4):507-13.

22. Parker G, Patterson A, Hadzi-Pavlovic D. Emotional response patterns of depression, grief, sadness and stress to differing life events. A quantitative analysis. J Affect Disorder. 2015;175:229-32. 
23. Sullivan - Bolyai S, Deatrick J, Grupps P, et al. Constant Vigilance: Mothers work parenting young children with type 1 diabetes. Journal of Pediatrics Nursing. 2003;18:21-9.

24. Weibe DJ, Gelfand D, Butler JM, et al. Longitudinal associations of maternal depressive symptoms, maternal involvement and diabetes management across adolescence. J Pediatr Psychol. 2011;36:837-46.

25. Hansen JA, Weissbrod C, Schwartz DD, et al. Parental involvement in pediatric type 1 diabetes fathers and mothers psychological functioning and disease management. Families systems health. 2012;30:47-59.

26. Whittemore R, Jasper SS, Chan A, et al. Psychological experience of parents of children with type 1 diabetes. A systematic mixed-studies review. The diabetes educator. 2012;38:562-71.

27. Carcone A, Ellis D, Naar-king S. Linking caregiver strain to diabetes illness management and health outcomes in a sample of adolescent in chronically poor metabolic control. J Dev Behav Pediatr. 2012;33:343-51.

\section{Tables}

Table 1: ASD, Diabetes and control by mother age, mother education and mother employment. 


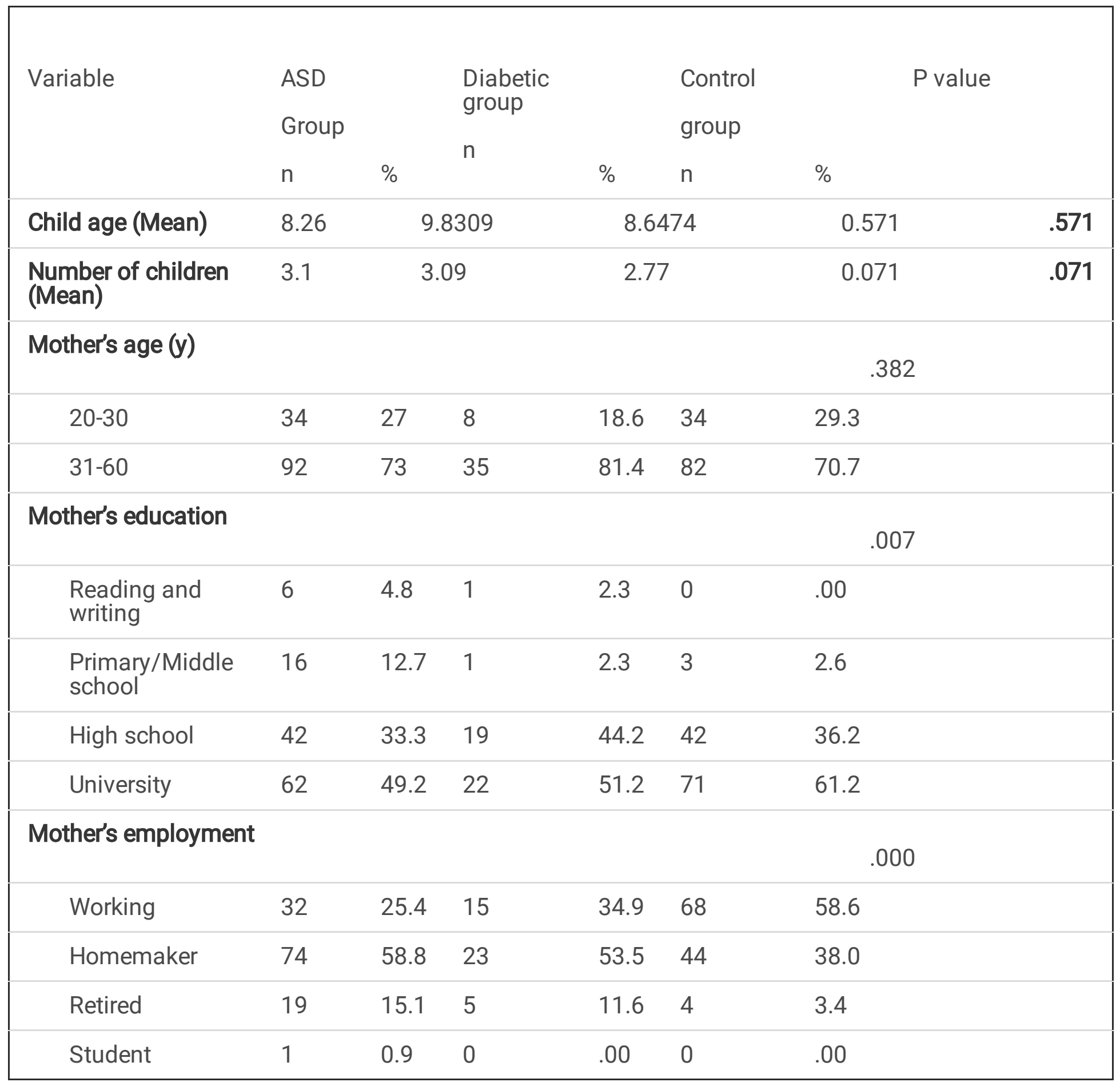

Table 2: DASS-21 and PSS-14 mean scores among diabetes group and controls. 


\begin{tabular}{|clllllll|}
\hline Instrument & $\begin{array}{l}\text { Diabetes group mean } \\
\text { score }\end{array}$ & $\begin{array}{l}\text { Controls mean } \\
\text { score } \\
\mathrm{N}=43\end{array}$ & $\mathrm{~N}=116$ & $\begin{array}{l}\mathrm{P} \\
\text { value }\end{array}$ & Odds & \multicolumn{2}{c|}{$\begin{array}{l}\text { 95\% Confidence } \\
\text { interval }\end{array}$} \\
\hline DASS-21 total & 41.58 & 29.81 & 0.019 & & & \\
\hline Depression & 10.81 & 9.34 & 0.395 & 1.148 & 0.568 & 2.318 \\
\hline Anxiety & 10.91 & 7.81 & 0.053 & 2.228 & 1.085 & 4.573 \\
\hline Stress & 14.05 & 12.66 & 0.404 & 1.324 & 0.645 & 2.717 \\
\hline PSS-14 & 28.63 & 25.87 & 0.003 & & & \\
\hline
\end{tabular}

Table 3: DASS-21 and PSS-14 mean scores among diabetes group and ASD group.

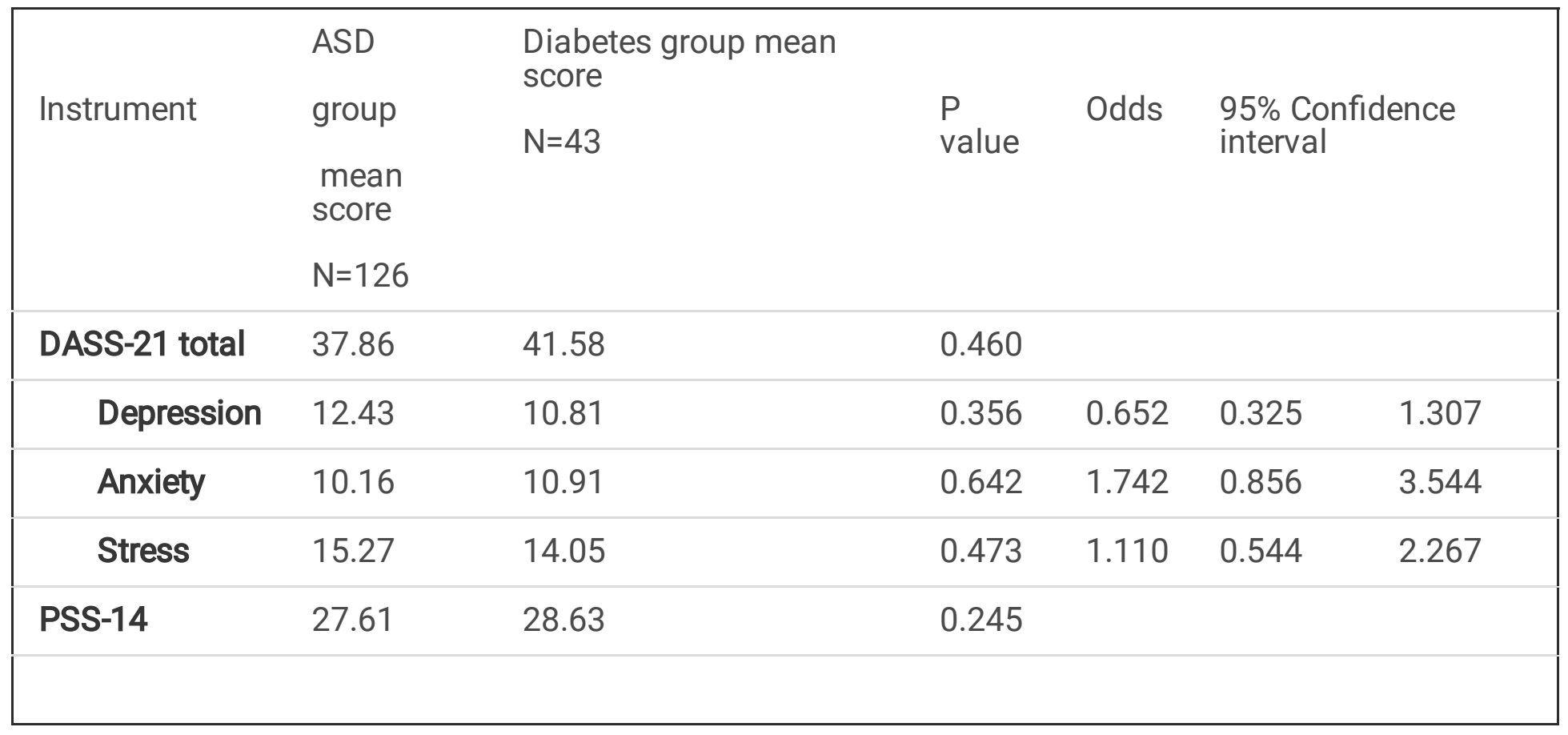

Table 4: DASS-21 and PSS-14 mean scores among ASD group, diabetes group and controls. 


\begin{tabular}{|cllll|}
\hline \multirow{2}{*}{ Instrument } & $\begin{array}{l}\text { ASD group } \\
\text { mean score }\end{array}$ & Diabetes group mean score N=43 & $\begin{array}{l}\text { Control } \\
\text { mean score }\end{array}$ & P value \\
& $\mathrm{N}=126$ & & $\mathrm{~N}=116$ & \\
\hline DASS-21 total & 37.86 & 41.58 & 29.81 & 0.015 \\
\hline Depression & 12.43 & 11.49 & 9.34 & 0.042 \\
\hline Anxiety & 10.16 & 12.70 & 7.81 & 0.007 \\
\hline Stress & 15.27 & 17.40 & 12.66 & 0.030 \\
\hline PSS-14 & 27.61 & 28.81 & 25.87 & 0.002 \\
\hline
\end{tabular}

Table 5: DAAS-21 PSS-14 Depression, anxiety and stress among mothers of case 1,2 and control

\begin{tabular}{|c|c|c|c|c|c|c|c|}
\hline \multirow[t]{2}{*}{ Instrument } & $\begin{array}{l}\text { Mothers } \\
\text { ASD } \\
n=126\end{array}$ & & \multicolumn{2}{|c|}{$\begin{array}{l}\text { Mothers Diabetes } \\
n=43\end{array}$} & \multicolumn{2}{|c|}{$\begin{array}{l}\text { Mothers Control } \\
n=116\end{array}$} & \multirow[t]{2}{*}{ P-value } \\
\hline & $\mathrm{N}$ & $\%$ & $N$ & $\%$ & $\mathrm{~N}$ & $\%$ & \\
\hline \multicolumn{8}{|l|}{ Dass-21 } \\
\hline \multicolumn{8}{|l|}{ Depression } \\
\hline Yes & 72 & 57.1 & 20 & 46.5 & 50 & 43.1 & \multirow[t]{2}{*}{0.046} \\
\hline No & 54 & 42.9 & 23 & 53.5 & 66 & 56.9 & \\
\hline \multicolumn{8}{|l|}{ Anxiety } \\
\hline Yes & 62 & 49.2 & 27 & 62.8 & 50 & 43.1 & \multirow[t]{2}{*}{0.034} \\
\hline No & 64 & 50.8 & 16 & 37.2 & 66 & 56.9 & \\
\hline \multicolumn{8}{|l|}{ Stress } \\
\hline Yes & 76 & 60.3 & 27 & 62.8 & 65 & 56 & \multirow[t]{2}{*}{0.009} \\
\hline No & 50 & 36.7 & 16 & 37.2 & 51 & 44 & \\
\hline Pss-14 & 27.61 & & & 28.63 & & 25.87 & 0.004 \\
\hline
\end{tabular}

